\title{
Pasado, presente y futuro del búfalo en Argentina
}

\author{
Crudeli, G.A.'; Patiño, E.M.2; Maldonado Vargas, P.'-3; Konrad, J.L. ${ }^{1}$
}

${ }^{1}$ Cátedra Teriogenología; ${ }^{2}$ Cátedra Tecnología de los Alimentos, ${ }^{3}$ Cátedra de Sociología Rural y Urbana, Facultad de Ciencias Veterinarias, UNNE, Sargento Cabral 2139, Corrientes (3400), Argentina,

Tel/Fax: 0379-4425753. Email: gacrudeli@hotmail.com

\begin{abstract}
Resumen
Crudeli, G.A.; Patiño, E.M.; Maldonado Vargas, P.; Konrad, J.L.: Pasado, presente y futuro del búfalo en Argentina. Rev. vet. 25: 2, 140-145, 2014. El búfalo comenzó a ser introducido en Argentina a comienzos del siglo XX, mediante la importación de razas Mediterránea, Murrah y Jafarabadi. En la actualidad la población bubalina es de 87.711 cabezas, distribuidas en 20 de las 23 provincias que componen el territorio nacional. El $80 \%$ de la población de búfalos se encuentra en el nordeste argentino, siendo las provincias de Formosa y Corrientes las que cuentan con las mayores densidades poblacionales. En el país existen 8 millones de hectáreas aptas para soportar una carga de 4 millones de búfalos.
\end{abstract}

Palabras clave: búfalo, población, Argentina.

\begin{abstract}
Crudeli, G.A.; Patiño, E.M.; Maldonado Vargas, P.; Konrad, J.L.: Past, present and future of buffalo in Argentina. Rev. vet. 25: 2, 140-145, 2014. The buffalo began to be introduced to the country in the early twentieth century, by importing Mediterranean, Murrah and Jafarabadi breeds. Today buffalo population reached 87.711 heads, which are distributed in 20 out of the 23 provinces that conforms the country. The $80 \%$ of the population of buffaloes is found in northeastern region, being the provinces of Formosa and Corrientes those with the largest buffalo population. The country has 8 million hectares suitable to withstand a load of 4 million buffaloes.
\end{abstract}

Key words: buffalo, population, Argentina.

\section{PASADO}

Los primeros búfalos llegaron a Argentina a principios del siglo XX, procedentes de Rumania y con destino a la provincia de Entre Ríos, donde se intentó cruzarlos con bovinos para destinarlos a la producción de leche ${ }^{2,3}$. Al fracasar esta experiencia los búfalos prácticamente desaparecieron, excepto algunos que fueron librados a la caza mayor.

Después de los fallidos intentos de cruzar el búfalo con el ganado bovino, al no haberse considerado la incompatibilidad cromosómica (el búfalo tiene 50 cromosomas y el bovino 60), debieron pasar varios años para que recién a comienzos de la década de 1980 se reanudaran las importaciones que en pequeña escala, fueron realizadas desde Italia, Brasil y Paraguay. Los ejemplares importados pertenecían a las razas Mediterráneo, Murrah y Jafarabadi ${ }^{2,8}$.

En 1979 se importaron 100 animales de la razas Jafarabadi y Murrah; 40 de ellos fueron enviados a la Estancia Santa Rosa ubicada en la localidad de Esquina, Provincia de Corrientes y los 60 restantes a las estancias

La Florencia y La Alicia en la localidad de San Cristóbal, provincia de Santa Fe ${ }^{4,7,8}$. En 1983 se creó la Asociación Argentina de Criadores de Búfalos (AACB) y en 1985 se registraron los primeros planteles puros abriéndose el primer libro de registros genealógicos ${ }^{4,8}$. En los años ' 90 se produjeron grandes importaciones, más de 5.000 vientres y reproductores seleccionados desde Brasil, lo cual consolidó la cría del búfalo en el país ${ }^{8}$.

En Argentina se crían tres de las razas de mayor importancia económica en el mundo: Mediterránea, que representa el $70 \%$ de la población bubalina del país, Murrah y Jafarabadi ${ }^{8}$, teniendo esta última escasa presencia en nuestro medio. Todas son de doble propósito, carne y leche, y a veces triple ya que en otros países se emplean para trabajo. En nuestro país los búfalos son empleados mayoritariamente para la producción de carne siendo incipiente la producción de leche. No son utilizados para trabajo. Pasemos revista a algunas características de estas razas:

Mediterránea: se formó en Italia. Están definidos como raza en Europa y demás zonas costeras del Mediterráneo. Sus colores comunes son el negro, gris oscuro, marrón oscuro y pizarra. Presenta cuernos medianos dirigidos hacia atrás y hacia los costados con las pun- 
Tabla 1. Existencia de búfalos en Argentina, por provincias y categorías.

\begin{tabular}{|c|c|c|c|c|c|c|c|c|c|c|}
\hline \multirow{2}{*}{ provincia } & \multicolumn{10}{|c|}{ Categoría } \\
\hline & $\mathrm{A}$ & $\mathrm{B}$ & $\mathrm{C}$ & $\mathrm{D}$ & $\mathrm{E}$ & $\mathrm{F}$ & G & $\mathrm{H}$ & I & total \\
\hline Formosa & 3 & 3.357 & 1.701 & 3.965 & 3.887 & 14 & 638 & 17.612 & 5.749 & 36.926 \\
\hline Corrientes & 3 & 831 & 1.160 & 2.523 & 2.508 & 102 & 932 & 12.039 & 3.299 & 23.397 \\
\hline Chaco & 6 & 620 & 830 & 895 & 789 & 20 & 465 & 3.313 & 1.470 & 8.408 \\
\hline Bs. Aires & 6 & 516 & 351 & 1.486 & 1.447 & 407 & 250 & 961 & 2.956 & 8.380 \\
\hline Entre Ríos & 2 & 582 & 367 & 560 & 436 & 6 & 156 & 1.346 & 1.167 & 4.622 \\
\hline Santa Fe & - & 75 & 96 & 205 & 162 & 3 & 83 & 671 & 290 & 1.585 \\
\hline Misiones & 10 & 100 & 51 & 241 & 174 & 2 & 55 & 624 & 158 & 1.415 \\
\hline Córdoba & - & 174 & 1 & 167 & 125 & 8 & 54 & 85 & 130 & 744 \\
\hline Sgo. del Estero & 5 & 63 & 30 & 104 & 74 & 34 & 75 & 209 & 147 & 741 \\
\hline La Pampa & - & - & 49 & 84 & 106 & 25 & 150 & 205 & 26 & 645 \\
\hline Neuquén & - & - & - & - & - & - & - & 2 & 230 & 232 \\
\hline San Luis & - & - & 15 & 16 & 20 & 6 & 6 & 72 & 8 & 143 \\
\hline Tucumán & 7 & 18 & 31 & 17 & 19 & - & 4 & 8 & 35 & 139 \\
\hline Mendoza & - & - & 20 & 1 & 13 & - & 35 & 50 & - & 119 \\
\hline La Rioja & - & 50 & - & - & - & - & 3 & - & - & 53 \\
\hline Salta & - & 23 & - & 4 & - & 2 & 18 & - & - & 47 \\
\hline Jujuy & - & - & 1 & 10 & 9 & - & 3 & 18 & - & 41 \\
\hline San Juan & - & - & 5 & 7 & 1 & - & 6 & - & 20 & 39 \\
\hline Catamarca & 26 & - & - & - & - & - & - & - & - & 26 \\
\hline Río Negro & - & - & - & - & 2 & - & 3 & 4 & - & 9 \\
\hline total & 68 & 6.409 & 4.708 & 10.285 & 9.772 & 629 & 2.936 & 37.219 & 15.685 & 87.711 \\
\hline
\end{tabular}

Fuente: SENASA (2014). Categorías: A: buey (buey búfalo), B: novillito (bubillito), C: novillo (bubillo), D: ternera (bucerra), E: ternero (bucerro), F: torito (butorito), G: toro (búfalo), H: vaca (búfala), I: vaquillona (bubilla).

tas cerradas hacia arriba y hacia adentro formando una media luna. Los adultos tienen un peso promedio de 700 a $800 \mathrm{~kg}$ en los machos y 600 a $650 \mathrm{~kg}$ en las hembras. El cuerpo es compacto, macizo, profundo, ancho en relación a su largo, con patas cortas y robustas ${ }^{1}$.

Murrah: su origen se encuentra en la región del Punjab, en el noroeste de la India. Murrah es una palabra hindú que significa espiralado y deriva de la forma de sus cuernos. Su color es negro azabache. Los cuernos son cortos, negros y espiralados desde su misma base, primero se orientan hacia los costados y luego completan la espiral hacia atrás. Es la raza más difundida en el mundo. Los adultos tienen un peso promedio de 600 a $800 \mathrm{~kg}$ en los machos y de 500 a $600 \mathrm{~kg}$ en las hembras ${ }^{1}$.

Jafarabadi: su nombre deriva de la ciudad del mismo nombre en la India. Son de color negro, aunque son aceptables manchas blancas en la cabeza y en la parte inferior de las patas. La frente es muy prominente. Tiene una cabeza grande, con una frente muy convexa de corte rectilíneo. Los cuernos son pesados, gruesos, fuertes y anchos, se orientan hacia abajo, atrás de los ojos, terminando con un rulo espiralado hacia atrás. Los machos tienen un peso de $700 \mathrm{a} 1500 \mathrm{~kg}$ y las hembras 650 a $900 \mathrm{~kg}$. Es la raza de mayor tamaño ${ }^{1}$.

Si bien la mayoría de los rodeos bubalinos existentes en Argentina fueron conformados originalmente con ejemplares puros de razas Mediterráneo, Murrah y Jafarabadi, debido a cruzamientos entre estas y al empleo de distintas genéticas lecheras bubalinas, se ha incrementado el número de mestizas de las dos prime- ras razas en nuestro país. Argentina posee actualmente la cuarta población bubalina del continente americano luego de Brasil, Venezuela y Colombia, encontrándose mayoritariamente concentrada en el subtrópico húmedo de la región del nordeste argentino (NEA), integrada por las provincias de Formosa, Corrientes, Chaco y Misiones.

En 2004, en la ciudad de Corrientes, se creó la Asociación para la Producción y el Desarrollo del Búfalo en la Argentina (ABUAR). Esta entidad fue el fruto de una mancomunidad entre productores de búfalos de la Provincia de Corrientes y docentes e investigadores de la Facultad de Ciencias Veterinarias de la Universidad Nacional del Nordeste (UNNE). En 2005 se estableció una cuota Hilton de 200 toneladas para la exportación de carne de búfalo al mercado de la Unión Europea, lo que permite comercializar con países de alto poder adquisitivo de Europa, como Holanda y Alemania ${ }^{5}$.

\section{PRESENTE}

De acuerdo a los datos oficiales aportados por el Servicio Nacional de Sanidad Animal (SENASA) la población de búfalos en Argentina en el año 2014 es de 87.711 cabezas ${ }^{6}$ (Tabla 1 ). De las 23 provincias que componen el territorio nacional existe población bubalina en 20 de ellas y solo en tres provincias del extremo sur argentino (Chubut, Santa Cruz y Tierra del Fuego) no se crían búfalos ${ }^{7}$.

Tomando en consideración la cantidad de búfalos que poseen las provincias argentinas, pueden ser agru- 
Tabla 2. Existencia de búfalos en la Provincia de Formosa, por departamentos y categorías.

\begin{tabular}{lcccccccccc}
\hline \multirow{2}{*}{ Departamentos } & \multicolumn{10}{c}{ Categoría } \\
\cline { 2 - 11 } & $\mathrm{A}$ & $\mathrm{B}$ & $\mathrm{C}$ & $\mathrm{D}$ & $\mathrm{E}$ & $\mathrm{F}$ & $\mathrm{G}$ & $\mathrm{H}$ & $\mathrm{I}$ & total \\
\hline Bermejo & - & - & - & - & 2 & - & 1 & 2 & - & 5 \\
Formosa & - & 829 & 438 & 2.112 & 1.870 & 5 & 409 & 7.662 & 2.835 & 16.160 \\
Laishi & - & 105 & 172 & 332 & 477 & 1 & 66 & 1.739 & 948 & 3.840 \\
Patiño & 3 & 152 & 65 & 137 & 108 & 7 & 19 & 670 & 283 & 1.444 \\
Pilagás & - & 978 & - & 496 & 524 & - & 67 & 4.058 & 858 & 6.981 \\
Pilcomayo & - & 389 & 242 & 480 & 489 & - & 33 & 1.737 & 414 & 3.784 \\
Pirané & - & 904 & 784 & 405 & 414 & 1 & 36 & 1.735 & 411 & 4.690 \\
Ramón Lista & - & - & - & 3 & 3 & - & 7 & 9 & - & 22 \\
total & 3 & 3.357 & 1.701 & 3.965 & 3.887 & 14 & 638 & 17.612 & 5.749 & 36.296 \\
\hline
\end{tabular}

Fuente: SENASA (2014). Categorías: A: buey (buey búfalo), B: novillito (bubillito), C: novillo (bubillo), D: ternera (bucerra), E: ternero (bucerro), F: torito (butorito), G: toro (búfalo), H: vaca (búfala), I: vaquillona (bubilla).

Tabla 3. Existencia de búfalos en la Provincia de Corrientes, por departamentos y categorías.

\begin{tabular}{|c|c|c|c|c|c|c|c|c|c|c|}
\hline \multirow{2}{*}{ Departamentos } & \multicolumn{10}{|c|}{ Categoría } \\
\hline & $\mathrm{A}$ & $\mathrm{B}$ & $\mathrm{C}$ & $\mathrm{D}$ & $\mathrm{E}$ & $\mathrm{F}$ & $\mathrm{G}$ & $\mathrm{H}$ & $\mathrm{I}$ & total \\
\hline Bella Vista & - & - & - & 7 & 15 & - & 1 & 22 & 20 & 65 \\
\hline Berón de Astrada & - & 3 & 1 & 131 & 82 & 9 & 28 & 451 & 152 & 857 \\
\hline Capital & - & 2 & 243 & 11 & 9 & - & 40 & 64 & 31 & 400 \\
\hline Concepción & - & 16 & 7 & 63 & 54 & - & 14 & 241 & 53 & 448 \\
\hline Curuzú Cuatia & - & 16 & 21 & 105 & 91 & - & 14 & 253 & 156 & 656 \\
\hline Empedrado & - & 110 & 5 & 470 & 389 & - & 46 & 1.648 & 278 & 2.946 \\
\hline Esquina & - & 140 & 47 & 108 & 117 & - & 47 & 167 & 38 & 664 \\
\hline General Paz & - & 269 & 531 & 545 & 593 & - & 257 & 4.196 & 1.432 & 7.823 \\
\hline Goya & - & 18 & - & - & 1 & - & 8 & 43 & 33 & 103 \\
\hline Itatí & - & 8 & 24 & 114 & 109 & - & 54 & 460 & 71 & 840 \\
\hline Ituzaingó & - & 71 & 12 & 203 & 133 & - & 105 & 841 & 306 & 1.671 \\
\hline Lavalle & - & 21 & - & 37 & 33 & - & 10 & 142 & 32 & 275 \\
\hline Mbucuruyá & - & 3 & 5 & 7 & 10 & - & 6 & 53 & 10 & 94 \\
\hline Mercedes & 1 & 84 & 146 & 218 & 216 & - & 80 & 580 & 169 & 1.494 \\
\hline Monte Caseros & - & - & 1 & 5 & 7 & - & 5 & 19 & 37 & 74 \\
\hline Paso de los Libres & - & 10 & 30 & 7 & 7 & - & 1 & 3 & 5 & 63 \\
\hline Saladas & 2 & 2 & 45 & 23 & 32 & - & 4 & 73 & 57 & 238 \\
\hline San Cosme & - & 3 & 1 & 59 & 58 & 1 & 13 & 266 & 31 & 432 \\
\hline San Luis del Palmar & - & 12 & 1 & 34 & 52 & - & 21 & 258 & 134 & 512 \\
\hline San Martín & - & 14 & 8 & 164 & 290 & - & 13 & 292 & 31 & 812 \\
\hline San Miguel & - & 22 & 18 & 84 & 88 & - & 77 & 1.303 & 122 & 1.714 \\
\hline San Roque & - & 1 & 3 & 44 & 52 & 5 & 14 & 116 & 39 & 274 \\
\hline Santo Tomé & - & 6 & 11 & 81 & 67 & 87 & 12 & 267 & 61 & 592 \\
\hline Sauce & - & - & - & 3 & 3 & - & 62 & 281 & 1 & 350 \\
\hline total & 3 & 831 & 1.160 & 2.523 & 2.508 & 102 & 932 & 12.039 & 3.299 & 23.397 \\
\hline
\end{tabular}

Fuente: SENASA. (2014). Categorías: A: buey (buey búfalo), B: novillito (bubillito), C: novillo (bubillo), D: ternera (bucerra), E: ternero (bucerro), F: torito (butorito), G: toro (búfalo), H: vaca (búfala), I: vaquillona (bubilla).

padas en: grandes, con más de 10.000 ejemplares ( 2 provincias); medianas, entre 1.000 y 10.000 ejemplares (5 provincias) y pequeñas, con menos de 1.000 ejemplares (13 provincias), como puede visualizarse en la Tabla 1.

Según categorías, la población bubalina del país se halla compuesta porcentualmente de la siguiente manera: búfalas $(42,4 \%)$; bubillas $(17,8 \%)$; bucerras $(11,7 \%)$; bucerros (11,1\%); bubillitos (7,3\%); bubillos (5,3\%); búfalos (3,3\%); butoritos $(0,7 \%)$ y buey búfalo $(0,4 \%)$ (Tabla 1$)$.

Las dos provincias consideradas con grandes poblaciones bubalinas son Formosa y Corrientes. Teniendo en cuenta la división del país por regiones, la del NEA (Formosa, Corrientes, Chaco y Misiones) concentra el $80 \%$ de la población bubalina del país. La provincia de Formosa es la que tiene la mayor cantidad de búfalos, con un registro oficial al año 2014 de 36.926 cabezas $^{6}$, ubicadas principalmente en los departamentos de Formosa, Pilagás y Pirané (Tabla 2). La zona cuenta con un total de 87 productores y existe un tambo de búfalas que produce quesos, yogurt y dulce de leche.

La Provincia de Corrientes es la segunda en cantidad de búfalos pero la primera en número de producto- 
Tabla 4. Existencia de búfalos en la Provincia de Chaco, por departamentos y categorías.

\begin{tabular}{|c|c|c|c|c|c|c|c|c|c|c|}
\hline \multirow{2}{*}{ Departamentos } & \multicolumn{10}{|c|}{ Categoría } \\
\hline & A & $\mathrm{B}$ & $\mathrm{C}$ & $\mathrm{D}$ & $\mathrm{E}$ & $\mathrm{F}$ & G & $\mathrm{H}$ & I & total \\
\hline 2 de Abril & - & 12 & - & - & - & - & 3 & 1 & - & 16 \\
\hline Almte. Brown & - & - & 2 & 7 & 9 & - & 16 & 54 & 11 & 99 \\
\hline Bermejo & - & 3 & 1 & 23 & 9 & - & 4 & 17 & 174 & 231 \\
\hline Cmte. Fernandez & - & - & - & - & - & - & 1 & 2 & - & 3 \\
\hline 12 de Octubre & - & - & 1 & - & - & - & - & - & - & 1 \\
\hline Gral. Belgrano & - & - & - & - & - & - & 13 & - & - & 13 \\
\hline Gral. Donovan & - & - & - & - & - & 3 & - & - & - & 3 \\
\hline Gral. Güemes & - & - & 2 & - & - & 1 & 2 & 12 & - & 17 \\
\hline Libertad & 6 & - & 10 & 48 & 52 & 2 & 17 & 195 & 120 & 450 \\
\hline Gral. San Martín & - & 12 & 22 & 68 & 69 & 1 & 26 & 361 & 70 & 629 \\
\hline Maipú & - & - & - & 20 & 27 & - & 3 & 22 & 10 & 82 \\
\hline 9 de Julio & - & - & 1 & - & - & - & - & - & - & 1 \\
\hline Pte. de la Plaza & - & - & - & - & - & 6 & - & - & - & 6 \\
\hline 1ro de Mayo & - & 1 & - & 26 & 35 & - & 7 & 38 & 25 & 132 \\
\hline Quitilipi & - & - & 2 & - & 3 & - & 1 & - & - & 6 \\
\hline San Fernando & - & 579 & 295 & 655 & 555 & - & 334 & 2.402 & 1.021 & 5.841 \\
\hline San Lorenzo & - & 2 & 259 & 28 & 12 & 5 & 4 & 57 & 7 & 374 \\
\hline Sgto. Cabral & - & 1 & 3 & - & - & 1 & 2 & 20 & - & 27 \\
\hline Tapenagá & - & 10 & 232 & 19 & 17 & 1 & 32 & 130 & 32 & 473 \\
\hline 25 de Mayo & - & - & - & 1 & 1 & - & - & 2 & - & 4 \\
\hline total & 6 & 620 & 830 & 895 & 789 & 20 & 465 & 3.313 & 1.470 & 8.408 \\
\hline
\end{tabular}

Fuente: SENASA. (2014). Categorías: A: buey (buey búfalo), B: novillito (bubillito), C: novillo (bubillo), D: ternera (bucerra), E: ternero (bucerro), F: torito (butorito), G: toro (búfalo), H: vaca (búfala), I: vaquillona (bubilla).

Tabla 5. Existencia de búfalos en la Provincia de Misiones, por departamentos y categorías.

\begin{tabular}{lcccccccccc}
\hline \multirow{2}{*}{ Departamentos } & \multicolumn{7}{c}{ Categoría } & \multicolumn{7}{c}{} \\
\cline { 2 - 9 } Apóstoles & $\mathrm{A}$ & $\mathrm{B}$ & $\mathrm{C}$ & $\mathrm{D}$ & $\mathrm{E}$ & $\mathrm{F}$ & $\mathrm{G}$ & $\mathrm{H}$ & $\mathrm{I}$ & total \\
Cainguás & - & - & 8 & 4 & 4 & - & 1 & 17 & - & 34 \\
Candelaria & - & - & - & - & - & - & 1 & - & - & 1 \\
Capital & - & - & - & - & - & - & 2 & 4 & - & 6 \\
Concepción & - & 9 & - & 15 & 6 & - & 8 & 80 & 2 & 120 \\
Eldorado & - & 8 & 8 & 2 & 3 & - & 2 & 11 & 6 & 40 \\
Gral. Belgrano & - & 1 & - & 4 & 3 & - & 1 & 12 & 6 & 27 \\
Guaraní & - & 24 & 15 & 93 & 52 & 1 & 15 & 155 & 46 & 401 \\
Iguazú & 2 & 28 & 11 & 13 & 15 & - & 4 & 128 & 13 & 214 \\
Leandro Alem & - & 3 & - & 3 & 2 & - & 1 & 18 & 4 & 31 \\
Libertador Gral. San Martin & 2 & - & - & 4 & 2 & - & 2 & 7 & 6 & 23 \\
Montecarlo & 4 & 1 & - & 36 & 33 & 1 & 8 & 67 & 13 & 163 \\
Oberá & - & 14 & - & 46 & 38 & - & 3 & 49 & 13 & 163 \\
San Ignacio & - & 1 & - & 2 & 2 & - & 1 & 8 & 1 & 15 \\
San Javier & - & - & - & 11 & 6 & - & 2 & 21 & 6 & 46 \\
25 de Mayo & 2 & 10 & 9 & 8 & 8 & - & 3 & 42 & 38 & 120 \\
total & - & 1 & - & - & - & - & 1 & 5 & 4 & 11 \\
\hline
\end{tabular}

Fuente: SENASA. (2014). Categorías: A: buey (buey búfalo), B: novillito (bubillito), C: novillo (bubillo), D: ternera (bucerra), E: ternero (bucerro), F: torito (butorito), G: toro (búfalo), H: vaca (búfala), I: vaquillona (bubilla).

res del país. Registra una población bubalina de 23.397 cabezas ${ }^{6}$ (Tabla 3) y 164 productores. Si se contabilizan los establecimientos con producción bubalina se superan los 200 ya que algunos productores presentan una o más unidades productivas en la provincia. Los departamentos con mayor población bubalina son los de General Paz, Empedrado y San Miguel.
Si se analizan los establecimientos ganaderos dedicados a la producción de búfalos de las provincias de Formosa y Corrientes, las cuales concentran la mayor población ganadera, es dable establecer en forma preliminar tres categorías diferenciadas por el número de animales que poseen. Así, la población se estratifica en pequeños productores (rango 1-50 cabezas), media- 
nos (rango 51-300) y grandes (más de 300). En trabajos posteriores debería establecerse la relación entre tales estratos con los objetivos empresariales y la tecnología adoptada.

En la Provincia de Formosa se aprecia que el 50\% de la población pertenece al estrato de grandes productores, en tanto que la Provincia de Corrientes solo presenta un $10 \%$ de productores de este estrato, concentrando el $90 \%$ de las existencias bubalinas en los estratos mediano y pequeño (Figura 1).

Merece destacarse el caso del establecimiento Guazú Cuá en Formosa, que dedica sus casi 40.000 hectáreas solo a albergar los aproximadamente 11.000 búfalos con que cuenta, lo cual representa cerca del $44 \%$ del rodeo provincial, y casi el $14 \%$ del rodeo nacional. Asimismo, es dable mencionar que el estado provincial cuenta con un rodeo de 504 animales cuyo fin es la difusión de la especie entre los distintos interesados, principalmente comunidades de menores recursos.

En la Provincia de Corrientes se debe mencionar que el establecimiento Santa Rosa Estancia, que marcara el desarrollo de la actividad bubalina en la región (Departamento Esquina) y llegara a concentrar una existencia de 10.000 búfalos, ha cambiado de actividad vendiéndose la totalidad de los animales.

La Provincia de Chaco es la tercera en cantidad de búfalos, registrando un total de 8.408 cabezas (41 productores), en establecimientos localizados mayoritariamente en el Departamento de San Fernando ${ }^{6}$ (Tabla 4).

Misiones es la séptima provincia tomando en cuenta la cantidad de búfalos, pues registra un total de 1.415 cabezas ${ }^{6}$ y 18 productores (Tejedor F., 2014, comunicación personal). El departamento General Belgrano es el que cuenta con mayor cantidad de búfalos (Tabla 5). Entre las provincias consideradas medianas por la cantidad de búfalos se deben mencionar a Buenos Aires (8.320 cabezas), Entre Ríos (4.622) y Santa Fe (1.585) ${ }^{6}$.

Cabe destacar que existen en el país cotos de caza que ofrecen búfalos de agua (Bubalus bubalis) para actividades cinegéticas, en las provincias de La Pampa, San Luis, Rio Negro, Buenos Aires, Entre Ríos, Córdoba, Neuquén, Formosa y Corrientes. Los búfalos desti-

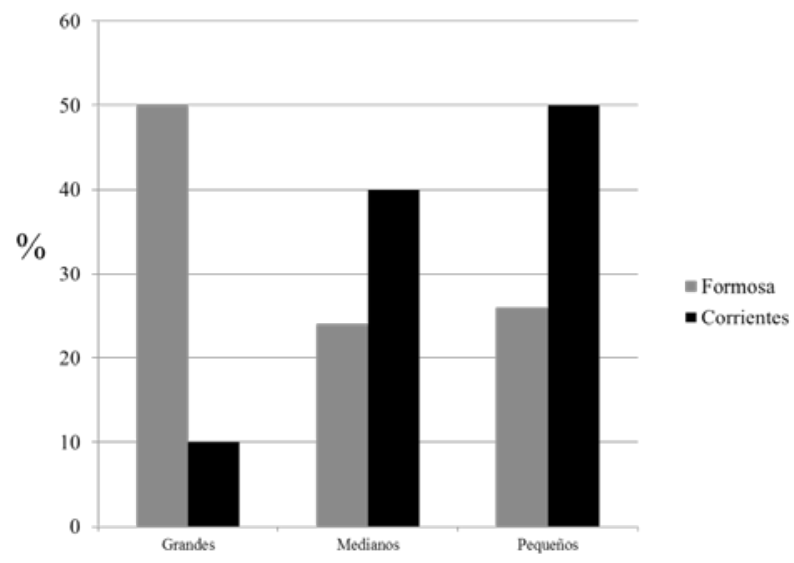

Figura 1. Porcentajes de productores de búfalos en las provincias de Corrientes y Formosa. nados a esta actividad son machos adultos de grandes cuernos que ya no operan como reproductores en los establecimientos bubalinos.

Actualmente la población bubalina de nuestro país debería ser mayor. No es así debido a dos motivos principales, el primero fue el fracaso de dos importaciones de miles de bubillas provenientes de Brasil en la década de 1990 debido a brotes de fiebre aftosa tanto en dicho país como en el nuestro. La otra causa es consecuencia de la ausencia de políticas ganaderas, lo que ocasiona que en Argentina una cantidad importante de bubillas del stock ganadero sean destinadas al mercado de carne, cuando deberían destinarse a la reposición de los establecimientos bubalinos.

De lo anteriormente manifestado se desprende la enorme importancia que tendría un programa de importación de semen desde Brasil o Italia, para una superación constante de la calidad de nuestros rodeos. En este sentido es muy largo el camino que aún se debe recorrer, ya que la calidad general de los ejemplares de nuestro país es inferior a la de aquellos de algunas regiones de Brasil y a la de todos los búfalos italianos.

\section{FUTURO}

En Argentina existe todavía una amplia región inexplotada o explotada ineficientemente desde el punto de vista pecuario, debido a la falta de adaptación del ganado vacuno a la misma, como son los sectores bajos e inundables que totalizan unas ocho millones de hectáreas. Tal superficie $\left(80.000 \mathrm{~km}^{2}\right)$ comprende 15.000 $\mathrm{km}^{2}$ de bajos submeridionales santafesinos; $10.000 \mathrm{~km}^{2}$ de costa de los ríos Paraná y Paraguay en Santa Fé, Chaco y Formosa; $20.000 \mathrm{~km}^{2}$ del pre-delta entrerriano y delta bonaerense; $15.000 \mathrm{~km}^{2}$ de esteros correntinos y $20.000 \mathrm{~km}^{2}$ de la cuenca del Río Salado en Buenos Aires ${ }^{8}$.

Esta colosal superficie es apta para soportar la carga de un búfalo cada dos hectáreas, lo que permitiría una población de 4 millones de búfalos, que no competirían con el ganado tradicional, el cual no es apto para estas zonas "marginales". La producción de búfalos en el país y principalmente en la región NEA presenta condiciones y ventajas competitivas para conformar un entramado o cluster productivo exitoso, ya que cuenta en su territorio con el ecosistema adecuado, también con el socio-sistema, es decir los productores dedicados a la actividad bubalina, y como tercer componente, existen instituciones de generación de tecnología como facultades de veterinaria y agencias del INTA, muchas de las cuales vienen dedicándose desde tiempo atrás a la investigación de temáticas referidas a la producción e innovación tecnológica para la producción bubalina, articulando y coordinando acciones con organismos provinciales y nacionales.

Resta plantear una agenda futura que priorice la dinámica de acción colectiva orientada a una visión compartida para la producción de búfalos en el país y en la región, con instrumentos, misiones colectivas y 
metas comunes para el crecimiento del capital social del entramado, entendido como la habilidad de los productores para trabajar conjuntamente en propuestas comunes, facilitando de esta manera la construcción de interacciones, rentas relacionales, adopción tecnológica, vínculos contractuales y una fuerte acción colectiva que lo posicione como un espacio propenso a la competitividad y a la innovación.

\section{REFERENCIAS}

1. Borghese A, Nazzu A. 2005. Buffalo population and strategies in the world. En: Búffalo, production and research. Publ. Instituto Esperimentale per la Zootecnia, FAO, Roma, p. 1-39.

2. Carrazoni, JA. 1998. El búfalo en el mundo y en nuestro país. Vet Arg 79: 2-7.
3. Incahusti D, Tagle ZC. 1980. Bovinotecnia (Cap. 9: Origen de los bovinos domésticos y bovideos), El Ateneo, Buenos Aires, p. 88-89.

4. Mastropolo R, Cravero J, Zava M. 1980. El búfalo. Rev Crea 85: 67-70.

5. Ministerio de Economía y Finanzas Públicas. 2005. Secretaria de Agricultura, Pesca y Alimentos. Exportaciones. Resolución 618/2005. http://infoleg.mecon.gov. ar/infolegInternet/anexos/105000-109999/108811/norma.htm.

6. Servicio Nacional de Sanidad Animal (SENASA). 2014. http://www.senasa.gov.ar.

7. Smaldone O. 1995. Cría de búfalos. Un establecimiento modelo. Rev Acaecer 227: 32-34.

8. Zava MA. 2001. El búfalo doméstico, Cap.14, Editora Orientación Gráfica, Buenos Aires, p. 521-596.

\section{Asociación Cooperadora de la Facultad de Ciencias Veterinarias Universidad Nacional del Nordeste}

Personería Jurídica No 647/92 y 912/00

Sargento Cabral 2139

3400 Corrientes

Argentina

La Asociación Cooperadora de la Facultad de Ciencias Veterinarias de la UNNE fue constituida el 10 de diciembre del año 1991 como entidad de bien público, con el objeto de promover y coadyuvar las actividades científicas, educativas y culturales relacionadas con las Ciencias Veterinarias. En tal sentido, implementa acciones para colaborar con la enseñanza, extensión, actualización y difusión científica que realiza dicha casa de estudios.

\section{Beneficios que brinda a sus asociados:}

- Fotocopias con descuentos especiales en la Fotocopiadora Copias.com que funciona dentro del predio de la Facultad de Ciencias Veterinarias.

- Descuentos para la adquisición de libros de distintas editoriales.

- Descuentos especiales en otros rubros.

Tel. (0379) 4425753 interno 186• Página Web www.vet.unne.edu.ar•E-mail: cooperadora@vet.unne.edu.ar 